\title{
Passive and active avoidance in the gerbil: Effects of sex and transfer of training
}

\author{
MICHAEL T. TWITTY and PETER F. GALVANI \\ State University of New York, Brockport, New York 14420
}

\begin{abstract}
The present experiment failed to obtain evidence of sex-related differences in either avoidance performance or activity levels in the Mongolian gerbil. Transfer of training between active and passive avoidance tasks was found, however, with negative transfer obtained in gerbils on a passive avoidance task following active shuttlebox avoidance acquisition, and positive transfer reported in animals in active avoidance learning following passive avoidance training. Possible reasons for these asymmetrical transfer effects were discussed.
\end{abstract}

It has generally been noted that female laboratory rats exhibit higher activity levels than male rats with respect to running-wheel performance, open-field activity, and exploratory behavior (see Denti \& Epstein, 1972). Denti and Epstein extended these findings by demonstrating (1) the superiority of female relative to male rats in the acquisition of an active avoidance response and (2) the superiority of males relative to females in a passive avoidance task. These authors concluded that the observed task-related performance differences were directly attributable to the sex-specific activity levels typically exhibited within this species.

In a similar fashion, the present study examined the relationships between sex differences and activity level in the gerbil on passive and active avoidance learning tasks. Presumably, if female gerbils are more active than males, then they should be superior in acquisition of an active avoidance response, but inferior in performance relative to males on a passive avoidance task where, by definition, activity must be inhibited (cf. Riddell, Galvani, \& Foster, 1976). In addition, transferof-training effects were evaluated. Subjects were required to first learn an active avoidance response, followed by the learning of a passive avoidance response, or vice versa. Presently, we know of no evidence as to whether or not the learning of a passive avoidance (or active avoidance) response will inhibit or facilitate the learning of the opposite task when a separate apparatus is employed for each of these avoidance procedures.

\section{METHOD}

\section{Subjects}

The subjects were 328 - to 9-month-old naive gerbils, 16 male and 16 female, obtained from Tumblebrook Farms, West Brookfield, Massachusetts. One week prior to the start of training, all subjects were individually caged in a continuously illum-

Reprint requests should be sent to Peter F. Galvani, Department of Psychology, State University College at Brockport, Brockport, New York 14420. inated room and maintained on ad-lib food and water. The subjects were randomly divided into two groups, each composed of eight males and eight females, which received either passive avoidance followed by active avoidance training (Group PA) or active avoidance followed by passive avoidance (Group AP).

\section{Apparatus}

The passive avoidance apparatus was an unpainted plywood box $(60 \times 40 \times 35 \mathrm{~cm}$ wide, inside dimensions) which surmounted a grid floor consisting of $.3-\mathrm{cm}$-diam stainless steel rods, spaced $1 \mathrm{~cm}$ apart and inserted into Plexiglas side rails. The platform was approximately $25 \mathrm{~cm}$ square, and was constructed of $1.25-\mathrm{cm}$-thick unpainted plywood. The platform was positioned so that two edges were flush against two inside walls of the box. An unscrambled 2.0-mA (nominal) $2.0-\mathrm{sec}$ electric shock was delivered to the grids through a GrasonStadler E6070B shock generator. Adjacent grid bars were of opposite polarity. Programming and data recording were accomplished by electromechanical equipment positioned next to the passive avoidance apparatus.

The active avoidance apparatus consisted of two Scientific Prototype Model A100S toggle-floor shuttleboxes (13.5 x $13 \times$ $40 \mathrm{~cm}$ ) with Plexiglas sides and tops and aluminum end walls. The shuttleboxes were divided in half by an aluminum insert with an arch-shaped cutout, $7 \mathrm{~cm}$ wide at the base $\times 9 \mathrm{~cm}$ in height at the center of the arch. The arch opening permitted passage from one side of the shuttlebox to the other. The shuttlebox floor consisted of stainless steel $.24-\mathrm{cm}$ grid bars, spaced $.95 \mathrm{~cm}$ apart, center to center. Each shuttlebox was enclosed in a ventilated sound-attentuated chamber illuminated by a 7.5-W incandescent lamp. A tonal CS $(2,500 \mathrm{~Hz})$ was delivered through an $8-\mathrm{cm}$ speaker, centered above each shuttlebox, via a BRS-Foringer audio generator (AU-902). The tone raised the ambient sound level ( $82 \mathrm{~dB}$ with ventilating fan on) about $2 \mathrm{~dB}$ on the $\mathrm{C}$ scale of a General Radio Company soundlevel meter, Type 1561-A. The US was a $.5-\mathrm{mA}$ (nominal) .5 -sec scrambled electric shock delivered to the shuttlebox floor by a Grason-Stadler Model GS700 shock generator. Programming and data recording equipment were located adjacent to the chambers housing the shuttleboxes.

\section{Procedure}

One week prior to the start of training, each subject was handled daily by the experimenter for about $3 \mathrm{~min}$. For the last 2 days of handling, each subject was transported from the animal colony room to the experimental rooms housing the avoidance apparatus. On Day 8 Group PA began 7 days of passive avoidance training which was followed by 2 days of active avoidance training. Passive avoidance training consisted of 4 habituation days on which the animal was placed on the 
platform, allowed to step down to a nonelectrified grid floor, and then removed from the apparatus. On each trial, the experimenter grasped the subject by the base of the tail and lowered it to the center of the platform with the subject's head oriented toward the corner of the box contiguous with the platform. On Day 5 the above procedure was repeated except that the subject received a 2.0 -sec 2.0 -mA shock upon stepping down onto the grid floor. Subjects were removed from the apparatus approximately $10 \mathrm{sec}$ following shock offset. Days 6 and 7, the test days, involved the same procedures as Days 1-4. Both activity time on the platform and step-down latency (SDL) were recorded each day. The SDL was the interval from the subject's placement on the platform until both forefeet contacted the grid. If the subject failed to step down off the platform in $180 \mathrm{sec}$, the trial was terminated and the subject's latency was recorded as $180 \mathrm{sec}$. The activity measure was recorded by the experimenter's activating a counter whenever the subject was observed to be active on the platform. Activity was defined as any locomotor behavior ranging from a single step to running about the platform, forward movement, backward movement, and turning around. It explicitly excluded behaviors such as postural changes, grooming, crouching, sitting, and lying down.

On Days 15 and 16 , following a 10-min adaptation period in the shuttlebox, Group PA gerbils received a single 100-trial acquisition session at a 30 -sec intertrial interval. Performance of a shuttle response during the 5-sec CS-US interval (a CR) resulted in immediate CS termination and US preclusion. On any given trial, failure to respond during the CS-US interval resulted in US presentation upon CS termination. Shuttle responses emitted at any time other than the CS-US interval (ITRs) were recorded as an index of activity.

Group AP received the identical handling procedures experienced by Group PA on Days 1-7, but order of training was reversed so that on Days 8 and 9 AP subjects received active avoidance training, followed on Days $10-16$ by passive avoidance training. Both groups were run simultaneously between 11:00 a.m. and 3:00 p.m. daily. Two separate replications ( $n=16$ each) were run. Note that for each group approximately $24 \mathrm{~h}$ separated the termination of the first training phase and the onset of the second training phase.

\section{RESULTS}

Male-female differences failed to emerge as a significant determinant of either acquisition or activity scores in either avoidance task. Moreover, no reliable interactions involving the sex variable were obtained for any measure. Hence, all analyses reported below, as well as graphic presentations of the results, have been collapsed across the sex variable. All statistical analyses and comparisons reported below employed $\alpha=.05$.

Figure 1 presents the mean percentage of time active on the platform over Days 1-7 for each group. Order of training did not appear to affect activity level on the early habituation days or on the passive avoidance tests (Days 6 and 7). Over Days 3-5, however, the PA subjects were clearly more active on the platform than the AP gerbils. The analysis of variance performed on these data indicated the effects of training order $[F(1,30)=6.94]$, days $[F(6,180)=10.68]$, and the Order by Days interaction $[F(6,180)=2.66]$ were all significant. Subsequent $t$ tests comparing activity scores on Days 5 and 6 indicated Group PA was reliably more active than Group AP before shock (Day 5) $[\mathrm{t}(30)=$

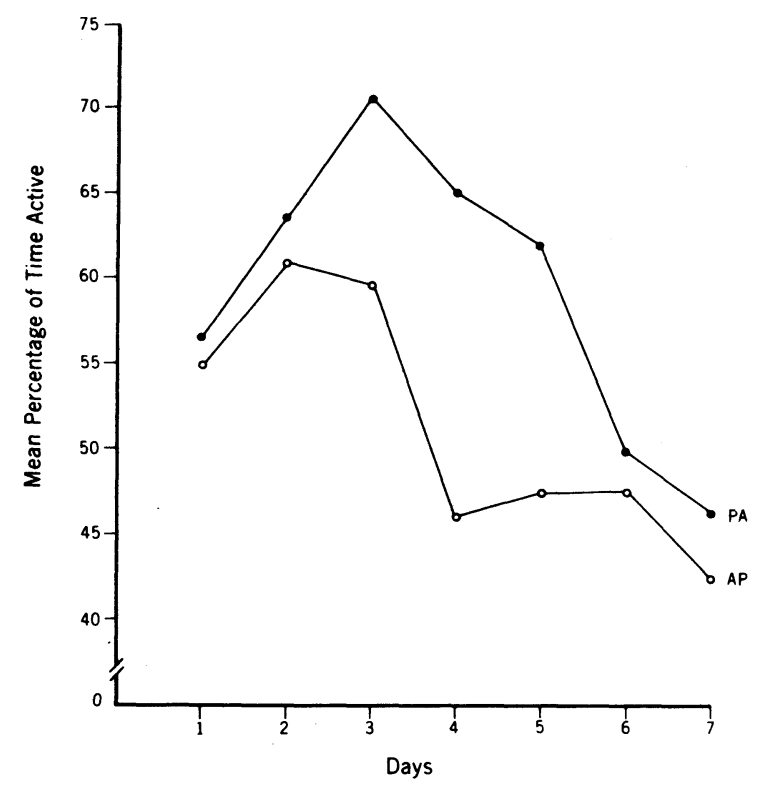

Figure 1. Mean percentage of time active on the safe platform in the passive avoidance situation, plotted for Days 1-7 for each group. (Group PA experienced passive avoidance training first; Group AP received active avoidance training first.)

2.74], but not after receipt of shock (Day 6). Apparently, the existence of the pre- but not the postshock differences in activity levels determined the Order by Days interaction.

The passive avoidance results are depicted in Figure 2 in terms of median SDL on Days 1-7 for each group. Since there was wide variability in latencies, nonparametric tests were employed (Wilcoxon matched-pairs signed-ranks test for within-subject comparisons and Mann-Whitney $U$ test for between-subject tests) in analyzing the SDL data. All tests were two-tailed.

Inspection of Figure 2 reveals both groups increased in SDL from Day 5 to Day 6 following punishment of the step-down response. Wilcoxon tests indicated these changes in SDL were significant for both Group PA $(\mathrm{T}=7, \mathrm{n}=16)$ and $\mathrm{AP}(\mathrm{T}=14, \mathrm{n}=16)$, thus demonstrating passive avoidance learning on Day 6 . On the subsequent passive avoidance test (Day 7), however, Group PA continued to passively avoid, while Group AP appeared to return to the preshock SDL baseline. Between-group comparisons revealed no difference in SDL on Day 6, but Group PA differed reliably from AP on Day $7\left(U=65, n_{1}\right.$ and $\left.n_{2}=16\right)$. This outcome suggests that passive avoidance learning was less persistent in gerbils previously trained in an active avoidance task relative to "naive" passive avoiders, that is, Group PA.

Figure 3 presents the mean CR and ITR probabilities for each group, over 10-trial blocks, for the first day of active avoidance. A 2 by 2 by 10 (order by response type by trial blocks) analysis of variance was performed on the CR and ITR data shown in Figure 3. The main effect of order was not significant $[F(1,30)=2.79]$; 


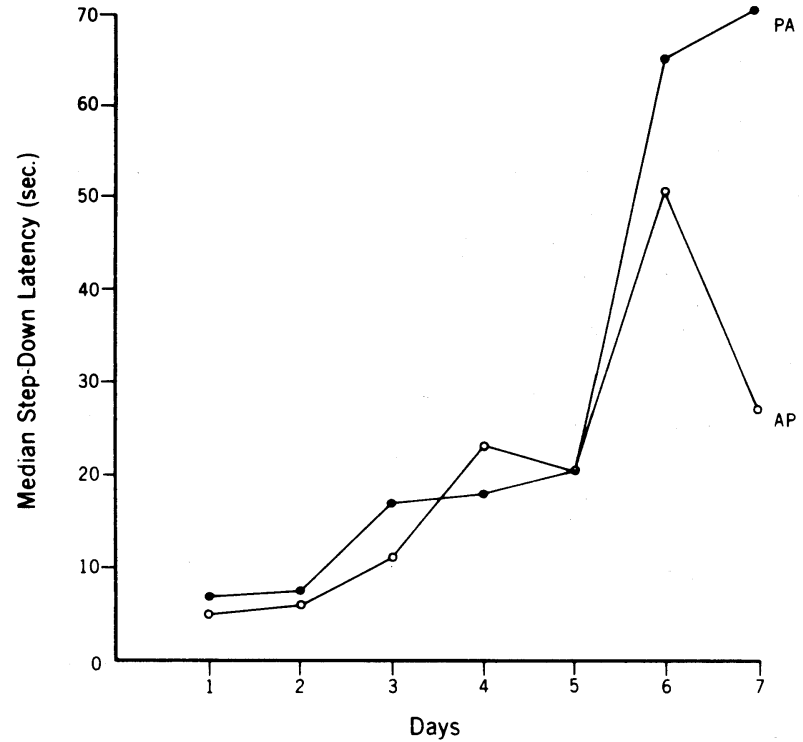

Figure 2. Median step-down latencies (SDL) in seconds plotted over Days 1-7 for each group.

however, there was a significant main effect of response type (CR vs. ITR) $[\mathrm{F}(1,30)=196.51]$ as well as of trial blocks $[F(9,270)=13.90]$. The following interactions were also significant: Trial Blocks by Order $[\mathrm{F}(9,270)=5.06]$, Trial Blocks by Response Type $[F(9,270)=31.57]$, and the triple interaction $[F(9,270)=2.40]$. Subsequent analyses revealed that for the ITR probability data there was no Trial Blocks by Order interaction $(\mathrm{F}<1)$; however, for the CR probability data the Trial Blocks by Order interaction was significant $[F(9,270)=9.46]$. The latter interaction presumably resulted from the cross-over in the CR probability curves of Groups PA and AP.
The results from the second day of active avoidance were not particularly informative. Generally, high performance levels were noted; 26 out of the 32 subjects made 85 or more CRs in the 100 -trial session.

\section{DISCUSSION}

Sex-related differences in avoidance performance previously observed in the rat (Denti \& Epstein, 1972) apparently failed to generalize across species to the Mongolian gerbil. Without exception, none of the indices of activity or learning indicated behavioral differences between male and female gerbils.

In terms of transfer-of-training effects, however, the influence of prior training was evident in both tasks. The passive avoidance activity data depicted in Figure 1 indicate greater locomotor activity in PA than in AP subjects on the 5 days prior to presentation of the aversive stimulus. The AP inferiority in activity level might suggest that prior exposure to the shuttlebox facilitated habituation to the "novel" passive avoidance situation. Alternatively, the lower activity level in Group AP might be due to inhibition mediated by a generalized expectancy of shock (or generalized fear) resulting from the AP gerbils' previous active avoidance training. Despite substantial differences in the two apparatus, both had grid floors which might have been critical environmental stimuli (given that shock was experienced through the grids) in mediating generalization of response tendencies and/or expectancies from one task to the other. Indirect support for such a speculation is provided by the reduction in platform activity in Group PA, to a level comparable to Group AP, following receipt of shock.

Although minimal platform activity should have been conducive to successful passive avoidance, that is, remaining on the platform, the SDL data indicated no differences between Groups PA and AP during Days 1-5. Apparently, platform activity was a more sensitive index of the influence of prior experience than the SDL measure, at least on the preshock days. Following punishment of the step-down response, however, passive avoidance was less persistent in Group AP than Group PA, with inferiority in performance reaching significance on Day 7

Presumably, the learning of active avoidance first adversely affected passive avoidance via generalization of the previously acquired tendency to take flight (shuttle) as a means of avoiding anticipated aversive stimulation. Once shock was received on

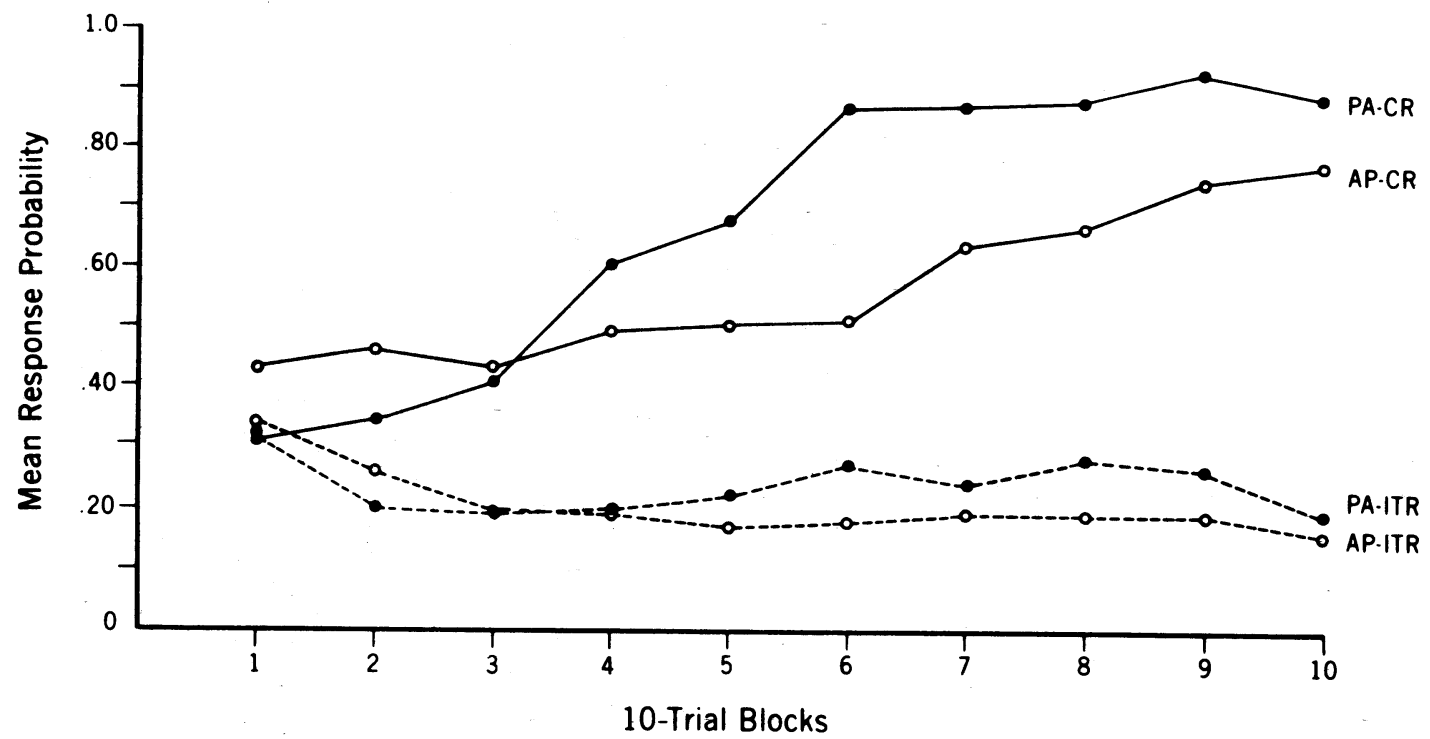

Figure 3. Mean conditioned response (CR) and intertrial response (ITR) probabilities for each group plotted over 10-trial blocks for Day 1 of shuttlebox avoidance training. 
Day 5, and fear and/or expectation of shock was established in the passive avoidance situation, the AP gerbils' performance might have been affected by competing tendencies to run that were not present in PA gerbils. Hence, negative transfer was obtained in the passive avoidance task.

In terms of active avoidance results, the ITR (activity) data showed no between-group differences. While ITR probability was relatively stable over trial blocks, CR probability increased over trials, providing clear evidence of learning in both groups. Transfer effects were evidenced in the CR probability data via the reliable Order by Trial Blocks interaction. Initially Group PA manifested slight negative transfer, but with continued training was clearly superior to Group AP. These results imply positive transfer from the passive to the active avoidance situation. Although the initial negative transfer in Group PA can be accounted for in terms of generalizing inhibition of movement from the passive avoidance task to the new aversive situation, the positive transfer effect was unexpected.

A tenuous hypothesis might interpret the positive transfer as indicating that the PA gerbils had acquired a general expectancy that aversive events were controllable via their behavior. When the initial attempt to passively avoid shocks in the shuttlebox failed, these subjects readily switched to a flight reaction in the presence of the CS. The AP gerbils, meanwhile, only gradually came to run in the presence of the CS, since they lacked the general expectation that shock was avoidable.

On this view, the asymmetrical transfer effects obtained in the present experiment (i.e., positive transfer in Group PA vs. negative transfer in Group AP) might be attributable to procedural differences in assessing transfer of training. For Group AP, following shock in the passive avoidance situation, transfer effects were assessed in the absence of further shocks. Thus, when AP animals stepped off the platform on Day 6, they were "reinforced" for an active response (shock was omitted). Accordingly, on Day 7 the active "flight" response competed more successfully with avoidance of the grid floor than on Day 6. Since transfer in PA gerbils was tested during active avoidance acquisition, on the other hand, their previously acquired avoidance behavior (immobility) was consistently punished.

Finally, two aspects of the present results, relative to previous results obtained in the Brockport laboratories, deserve comment. First, Riddell et al. (1976, Experiment 1) found male gerbils superior to females in passive avoidance acquisition in a task requiring escape onto a safe platform. In a passive avoidance retention test comparable to that of the present study, however, these authors subsequently found no sex differences in performance as reported herein. Second, platform activity levels shown in Figure 1 are substantially below those previously reported in Galvani, Riddell, and Foster (1975). The present activity measure was essentially an indicant of locomotor activity, specifically excluding postural changes which were included in the activity measure in Galvani et al. Despite differences in activity levels attributable to these differences in the definition of activity, both studies found dramatic postshock activity decrements.

\section{REFERENCES}

Denti, A., \& Epstein, A. Sex differences in the acquisition of two kinds of avoidance behavior in rats. Physiology and Behavior, 1972, 8, 611-615.

Galvani, P. F., Riddell, W. I., \& Foster, K. M. Passive avoidance in rats and gerbils as a function of speciesspecific exploratory tendencies. Behavioral Biology, 1975 , 13, 277-290.

Riddell, W. I., Galvani, P. F., \& Foster, K. M. The role of escape-motivated behavior in aversive conditioning in rats and gerbils. Behavioral Biology, 1976, 17, 485-494.

(Received for publication April 28, 1977.) 